\title{
SiGe double barrier resonant tunneling diodes on bulk SiGe substrates with high peak-to-valley current ratio
}

\author{
S. Tsujino ${ }^{\mathrm{a})}$ \\ Laboratory for Micro- and Nanotechnology, Paul Scherrer Institut, CH-5200 Villigen-PSI, Switzerland \\ N. Usami ${ }^{\mathrm{b})}$ \\ Institute for Materials Research, Tohoku University, 2-1-1 Katahira, Aoba-ku, Sendai 980-8577, Japan
}

A. Weber, G. Mussler, V. Shushunova, and D. Grützmacher

Laboratory for Micro- and Nanotechnology, Paul Scherrer Institut, CH-5200 Villigen-PSI, Switzerland

Y. Azuma and K. Nakajima

Institute for Materials Research, Tohoku University, 2-1-1 Katahira, Aoba-ku, Sendai 980-8577, Japan

(Received 7 May 2007; accepted 18 June 2007; published online 19 July 2007)

\begin{abstract}
The authors present high-quality $p$-type SiGe double barrier resonant tunneling diodes obtained from bulk SiGe substrates grown by the multicomponent zone-melting method and by the layer deposition with molecular beam epitaxy. Devices exhibit a high peak-to-valley current ratio up to 8.8 at $4.2 \mathrm{~K}$ and a negative differential resistance up to $340 \mathrm{~K}$. The result demonstrates that bulk $\mathrm{SiGe}$ substrates have a clear potential impact for fabricating high-performance SiGe heterostructure devices based on quantum transport. (C) 2007 American Institute of Physics.
\end{abstract}

[DOI: $10.1063 / 1.2756363$ ]

The multicomponent zone-melting (MCZM) method has been developed to grow multicomponent semiconductor bulk crystals with arbitrary compositions. By this method, the growth temperature can be kept constant and the depletion of one constituent component can be prevented. ${ }^{1,2}$ The method is applicable for crystals such as SiGe (Ref. 3) and InGaAs (Ref. 4) which retain complete solubility in both liquid and solid states. In contrast with the bulk substrates grown by conventional methods, ${ }^{5-10}$ our homemade multicomponent semiconductor substrates consist of single crystal domains with uniform compositions and with the size of a few millimeters to centimeters in diameter and are almost free from microscopic structural imperfections. ${ }^{11}$ These characteristics make our substrates applicable to device developments. To test the quality of bulk SiGe crystals with a high Ge content, we deposited double barrier resonant tunneling diodes (RTDs) by low-temperature molecular beam epitaxy (MBE) on (100)-oriented bulk $\mathrm{Si}_{1-x_{s}} \mathrm{Ge}_{x_{s}}$ substrates with $x_{s}$ equal to $\sim 0.9$ and characterized the resonant tunneling properties at various temperatures.

We have studied three RTD samples A, B, and C. The layer sequence is shown in Fig. 1(a). RTDs A and B consist of a $\mathrm{Si}_{1-x} \mathrm{Ge}_{x}$ quantum well with thicknesses equal to 2.5 and $3.5 \mathrm{~nm}$, respectively. The Ge content $x$ of the quantum well, spacer layers, and contact layers was equal to 0.93 (see below), and the Ge content of the barriers was equal to 0.3. The quantum well of RTD C is a 3.5-nm-thick Ge layer. The substrates were semicircle shaped with diameter equal to $\sim 15 \mathrm{~mm}$. These were obtained from a bulk crystal grown by using a $\mathrm{Ge}(100)$ seed crystal, a polycrystalline Ge for melt, and a $\mathrm{Si}(100)$ crystal to feed $\mathrm{Si}$ to the melt. ${ }^{12}$

The wafer surface of the SiGe substrates were polished by the chemical-mechanical polishing process and chemically treated in $\mathrm{NH}_{4} \mathrm{OH} / \mathrm{H}_{2} \mathrm{O}_{2}$ solution. Prior to the $\mathrm{MBE}$

\footnotetext{
${ }^{a)}$ Electronic mail: soichiro.tsujino@psi.ch

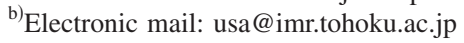

growth, substrates were again chemically cleaned and followed by annealing in vacuum at $800{ }^{\circ} \mathrm{C}$ for $15 \mathrm{~min}$. The double barrier structures were then deposited at $300{ }^{\circ} \mathrm{C}$. The deposition rates of $\mathrm{Si}_{1-x} \mathrm{Ge}_{x}$ layers and of $\mathrm{Si}_{1-y} \mathrm{Ge}_{y}$ barriers were equal to 0.7 and $1.43 \AA / \mathrm{s}$, respectively. $40 \mathrm{~s}$ long growth interruptions were inserted at the interfaces between $\mathrm{Si}_{1-x} \mathrm{Ge}_{x}$ layers and $\mathrm{Si}_{1-y} \mathrm{Ge}_{y}$ layers for the stabilization of the growth rate after the change of the $\mathrm{Si}$ and the Ge flux. $p^{+}-\mathrm{Si}_{1-x} \mathrm{Ge}_{x}$ contact layers were doped by coevaporation of boron at the concentration equal to $1.2 \times 10^{18} \mathrm{~cm}^{-3}$. From the reciprocal space map in the (224) direction, we found that the deposited layers are grown commensurately on the SiGe bulk substrates. The Ge content of the substrate $x_{s}$ and that of the deposited layer (average value) $x$ were determined by (004) x-ray diffraction; we obtained that $x_{s}$ is equal to $0.88 \pm 0.02$ and $x$ is equal to $0.93 \pm 0.02$.

From these samples, square-shaped diodes were fabricated. At first, top Al electrodes were patterned by photolithography and lift-off. The sizes of the mesas are 30, 20, 10, and $6 \mu \mathrm{m}$. Secondly, using the $\mathrm{Al}$ electrodes as the mask, the top-contact layer and the double-barrier structure were patterned by reactive ion etching (RIE) using $\mathrm{SF}_{6}$ gas. Thirdly, the $\mathrm{Al}$ electrodes were patterned on to the exposed bottom contact layer. Fourthly, 500-nm-thick $\mathrm{SiN}_{x}$ insulation layer was deposited at $300{ }^{\circ} \mathrm{C}$ by using plasma enhanced chemical vapor deposition. Finally, through holes to the top and the bottom Al electrodes were patterned by photolithography and RIE. Throughout the process, extreme care has been taken to minimize the temperature gradient across the sample substrates to avoid strain relaxation. The processed devices were mounted on ceramic chip carriers. After the wire bonding, the chip carriers were mounted on a variable temperature insert for measurement.

In Fig. 2, two-terminal current-voltage characteristics of $6 \mu \mathrm{m}$ square mesa devices measured at three different temperatures are shown. Nearly symmetric current-voltage characteristics were observed at all temperatures. For B, total of 
(a)

\begin{tabular}{|c|}
\hline $\mathrm{p}^{+} \mathrm{Si}(1-\mathrm{x}) \mathrm{Ge}(\mathrm{x}) 200 \mathrm{~nm}$ \\
\hline $\mathrm{Si}(1-x) \mathrm{Ge}(\mathrm{x}) 50 \mathrm{~nm}$ \\
\hline $\mathrm{Si}(1-\mathrm{y}) \mathrm{Ge}(\mathrm{y}) 4.0 \mathrm{~nm}$ \\
\hline Quantum well \\
\hline $\mathrm{Si}(1-\mathrm{y}) \mathrm{Ge}(\mathrm{y}) 4.0 \mathrm{~nm}$ \\
\hline $\mathrm{Si}(1-\mathrm{x}) \mathrm{Ge}(\mathrm{x}) 50 \mathrm{~nm}$ \\
\hline $\mathrm{p}^{+} \mathrm{Si}(1-\mathrm{x}) \mathrm{Ge}(\mathrm{x}) 400 \mathrm{~nm}$ \\
\hline Bulk Si $\left(1-x_{s}\right) G e\left(x_{s}\right)$ subst. \\
\hline
\end{tabular}

(b)

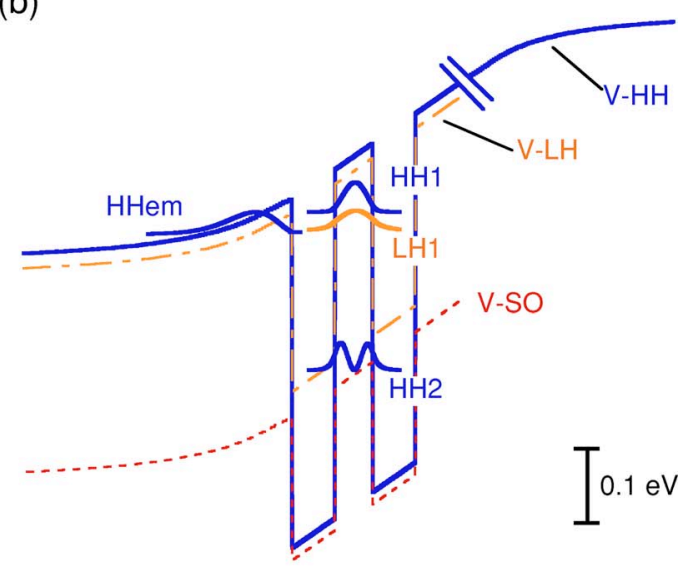

FIG. 1. (Color online) (a) Layer sequence of the $p$-SiGe double barrier resonant tunneling diode (RTD) structures on bulk SiGe substrates. RTDs A and B consist of 2.5-nm-thick and 3.5-nm-thick $\mathrm{Si}_{1-x} \mathrm{Ge}_{x}$ quantum well, respectively, with 4-nm-thick $\mathrm{Si}_{1-y} \mathrm{Ge}_{y}$ barriers clad by $p^{+}-\mathrm{Si}_{1-x} \mathrm{Ge}_{x}$ contact layers, where $x$ is equal to 0.93 and $y$ is equal to 0.3. RTD C consists of a 3.5-nm-thick Ge well. The Gecontent $x_{s}$ of the bulk substrate is equal to 0.88. (b) Schematic band diagram of RTD B calculated at the bias equal to $0.9 \mathrm{~V}$. Only representative states are shown by solid curves. HHn $(n=1,2)$ are the two highest heavy-hole (HH) states, HHem is the highest emitter state originated from the $\mathrm{HH}$ band, and LH1 is the highest light-hole (LH) state. $\mathrm{V}-\mathrm{HH}, \mathrm{V}-\mathrm{LH}$, and V-SO denote the band edge of the HH band, the LH band, and the split-off (SO) band, respectively, including the effect of strain.
20 devices with various sizes were tested. Among those, nine devices show nearly identical current-voltage characteristics, as the one in Fig. 2 (see also Fig. 3). For A and B, two resonances were observed at low temperatures in both polarities. These are ascribed to the resonant tunneling via the first two quantized states in the quantum well assigned as the first heavy-hole $(\mathrm{HH})$ state $\mathrm{HH} 1$ and the first light-hole (LH) state LH1. The assignment is consistent to a self-consistent 6-band $\mathbf{k} \cdot \mathbf{p}$ theory [Fig. 1(b)]. ${ }^{13}$ The precise shape and the oscillation of the current at the bias beyond LH1 resonances are affected by extrinsic and intrinsic bistabilities which have been observed for RTDs with large negative differential resistance (NDR). ${ }^{14}$ Detailed comparison of experiment with theory, including the bias- and temperature-dependent accumulation profiles at the emitter, the in-plane momentum dependent tunneling rate, and the nonequilibrium hole transport, is out of the scope of this letter. Here, we point out that the above peak assignment is consistent with the fact that the two peaks move to higher bias (confinement shift) by decreasing the well thickness from $3.5 \mathrm{~nm}$ (B) to $2.5 \mathrm{~nm}$ (A). The assignment is also consistent with the observation that the separation between the two peaks increases by the increase of $\mathrm{Ge}$ content and compressive strain in the well from $\mathrm{Si}_{0.07} \mathrm{Ge}_{0.93}$ (B) to $\mathrm{Ge}(\mathrm{C})$. The larger asymmetry of the current-voltage characteristic in $\mathrm{C}$ is likely due to partial islanding in the Ge well enhanced by the strain. ${ }^{15,16}$

For B shown in Fig. 2(b), we found that the peak-tovalley current ratio (PVR) of the LH1 peak at $4.2 \mathrm{~K}$ amounts to 8.0 in negative bias and 7.8 in positive bias. Figure 3 summarizes the LH1 peak properties of B for nine devices at $4.2 \mathrm{~K}$. The highest PVR equal to 8.8 was observed in a $30 \mu \mathrm{m}$ square mesa device. The peak current divided by nominal device area is approximately constant for each polarity. There is a slight tendency that the valley current per area increases with the decrease of the device size. This indicates that leak current through defects, for example via the sidewall of the etched mesa, partly limits the PVR. Nevertheless, these PVR values are the highest for the resonant tunneling via quasi-two-dimensional states in $p$-type $\mathrm{SiGe}$ RTDs. The highest PVR for a resonant tunneling through the quasi-two-dimensional states in $p$-type SiGe quantum well previously reported in literature is equal to $\sim 5$ observed at $4.2 \mathrm{~K}$ in $6 \mu \mathrm{m}$ diameter $\mathrm{Si}_{0.2} \mathrm{Ge}_{0.8} / \mathrm{Si}$ RTDs on $\mathrm{Si}_{0.5} \mathrm{Ge}_{0.5}$ virtual substrates. ${ }^{17}$ The highest reported PVR in $p$-type SiGe

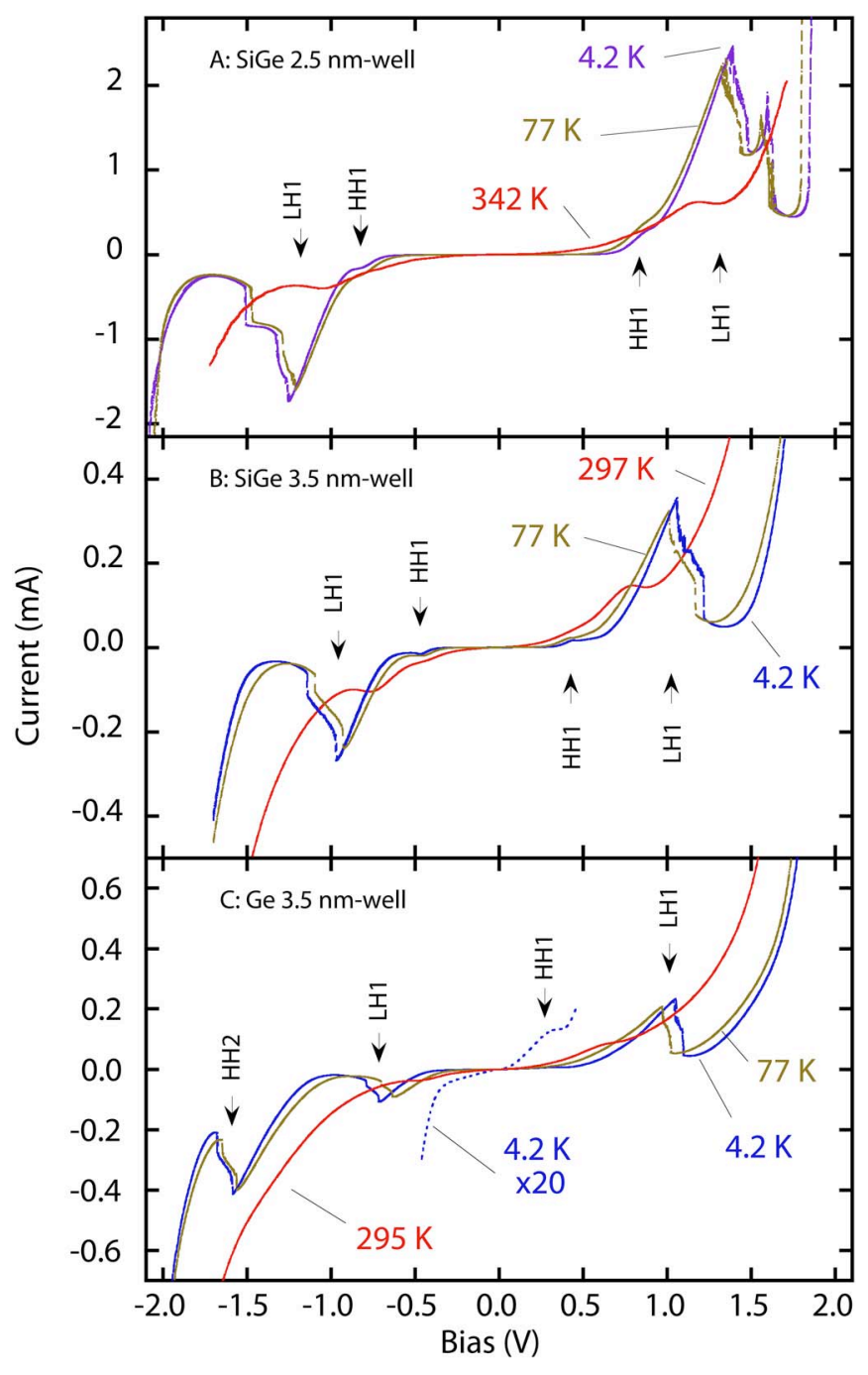

FIG. 2. (Color online) Current-voltage characteristics of three SiGe RTDs with $6 \mu \mathrm{m}$ square mesa measured at 4.2, $77 \mathrm{~K}$, and near room temperature. For $\mathrm{A}$ and $\mathrm{B}$, two resonances assigned as $\mathrm{HH}_{1}$ and $\mathrm{LH}_{1}$ are observed at low temperatures in both polarities. For $\mathrm{C}, \mathrm{HH}_{1}$ peak is observed only at the lowest temperature and in positive bias, and $\mathrm{HH}_{2}$ is also observed in negative bias. A record PV current ratio of 8.0 is observed in RTD B at $4.2 \mathrm{~K}$ in negative polarity. The negative differential resistance of the $\mathrm{LH}_{1}$ peak remains at and above room temperature for $\mathrm{A}$ and $\mathrm{B}$. 


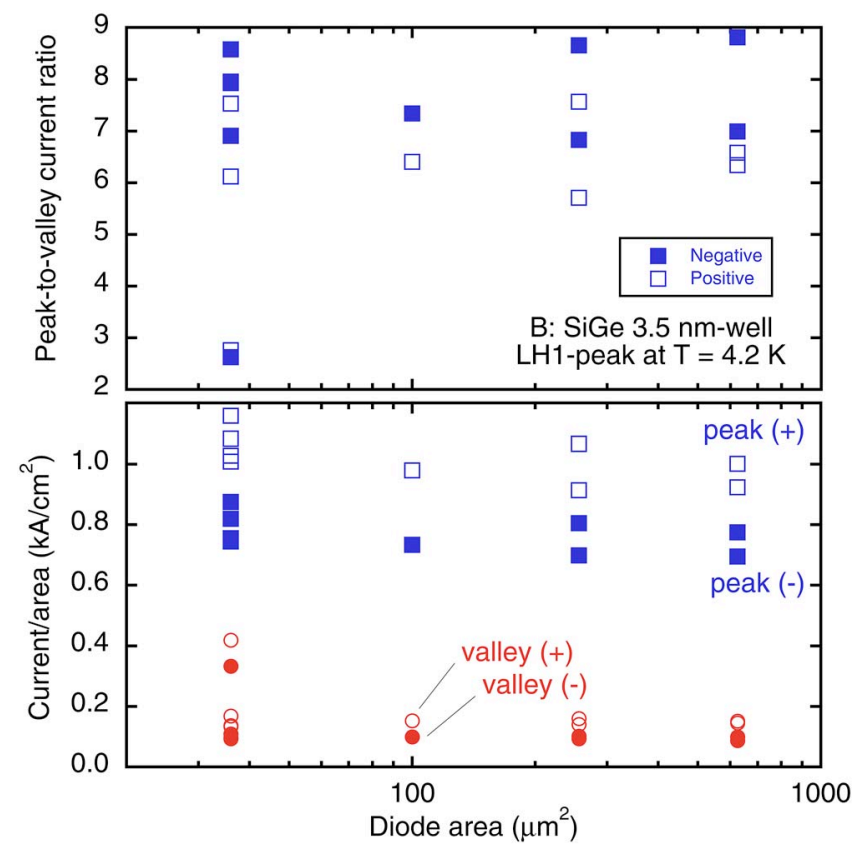

FIG. 3. (Color online) Summary of the resonant tunneling characteristics of LH1 peak of RTD B obtained from nine devices with various mesa sizes measured at $4.2 \mathrm{~K}$. The top panel shows the peak-to-valley current ratios. The bottom panel shows the peak and valley current divided by device area for positive (+, empty signs) and negative (-, filled signs) polarities.

RTDs is equal to 12 , which was observed at $11 \mathrm{mK}$ in a $480 \mathrm{~nm}$ diameter $p$ - $\mathrm{Si}_{0.83} \mathrm{Ge}_{0.17} / \mathrm{Si} \mathrm{RTD}$; however, this resonance is not likely due to the tunneling via the twodimensional states but is rather ascribed to the tunneling through zero-dimensional states or defects. ${ }^{18}$

Figure 2 also shows that the NDR of the LH1 peak remains at $297 \mathrm{~K}$ for B and up to $342 \mathrm{~K}$ for A. Above $77 \mathrm{~K}$, NDR decreased rapidly because of the increase of the valley current $I_{v}$ as well as the decrease of the peak resonant current $I_{p}$. PVR in negative bias decreased to two at $255 \mathrm{~K}$ for A and at $192 \mathrm{~K}$ for $\mathrm{B}$, respectively (not shown). $I_{v}$ is increased by thermally assisted tunneling via excited states and thermal excitation of holes over the barriers. ${ }^{19}$ Hence, to suppress the temperature-induced increase of $I_{v}$, the use of higher barriers will be required. ${ }^{20}$ On the other hand, more than a factor of 2 reduction of $I_{p}$ is due to the temperature-induced increase of the quasi-Fermi energy in the hole accumulation region in the emitter. ${ }^{21}$ Therefore, this can be improved by optimization of the device structure such as the concentration and profile of the contact doping.

The observed stable operation, especially the high PVR, is ascribed to the low defect density of our SiGe bulk substrates and the consecutive high-quality heterostructures. The low defect density enhances the NDR by minimizing the background current due to nonresonant current paths and the level broadening of the quantum states. Therefore, compared with virtual substrates, i.e., strain-relaxed thick films on commercially available Si or Ge substrate, the lack of defects such as threading dislocations and the concomitant fluctuation of the lattice orientation in submicrometer scale appears to be an advantage of our SiGe bulk substrates for fabricating high-performance heterostructure devices based on quantum transport.

In summary, we demonstrated the excellent quality of high Ge-content SiGe substrates grown by MCZM by means of the temperature-dependent electrical transport characteristics of RTDs deposited by MBE. Our results indicate that PVR at room temperature can be further improved by optimization of the device structure and the device processing. It should be remarked that an efficient resonant tunneling, as is demonstrated here, is a prerequisite to achieve lasing in SiGe electroluminescence devices such as quantum cascade emitters. ${ }^{22,23}$ Such RTDs also have a potential for the realization of Si-based quantum functional devices and circuits at room temperature. ${ }^{24}$

The authors acknowledge U. Gennser for helpful discussions and H. Sigg for careful reading of this letter. This work was supported in part by the Swiss National Science Foundation, by Grant-in-Aids for Scientific Research (S) No. 14102020 from Japan Society for the Promotion of Science, and by Industrical Technology Research Grant Program from NEDO of Japan.

${ }^{1}$ T. Suzuki, K. Nakajima, T. Kusunoki, and T. Katoh, J. Electron. Mater. 25, 357 (1996).

${ }^{2}$ K. Nakajima, S. Kodama, S. Miyashita, G. Sazaki, and S. Hiyamizu, J. Cryst. Growth 205, 270 (1999).

${ }^{3}$ R. W. Olesinski and G. J. Abbaschian, Bull. Alloy Phase Diagrams 5, 180 (1984).

${ }^{4}$ G. B. Stringfellow, Phase Equilibria Diagrams (American Ceramic Society, Columbus, OH, 1992), Vol. 9, p. 135.

${ }^{5}$ P. S. Dutta, J. Cryst. Growth 275, 106 (2005), and references therein.

${ }^{6}$ K. Nakajima, Y. Azuma, N. Usami, G. Sazaki, T. Ujihara, K. Fujiwara, T. Shishido, Y. Nishijima, and T. Jusunoki, Int. J. Mater. Prod. Technol. 22, 185 (2005), and references therein.

${ }^{7}$ Y. Hayakawa, T. Ozawa, T. Araki, M. Haris, and M. Kumagawa, J. Cryst. Growth 275, e421 (2005).

${ }^{8}$ M. Yildiz, S. Dost, and B. Lent, J. Cryst. Growth 280, 151 (2005).

${ }^{9}$ I. Yonenaga, J. Cryst. Growth 275, 91 (2005).

${ }^{10}$ K. Otsubo, H. Shoji, T. Kusunoki, T. Suzuki, T. Uchida, Y. Nishijima, and H. Ishikawa, IEEE Photonics Technol. Lett. 10, 1073 (1998).

${ }^{11}$ N. Usami, Y. Nose, K. Fujiwara, and K. Nakajima, Appl. Phys. Lett. 88, 221912 (2006).

${ }^{12}$ Y. Azuma, N. Usami, T. Ujihara, K. Fujiwara, Y. Murakami, and K. Nakajima, J. Cryst. Growth 250, 298 (2003).

${ }^{13}$ S. Tsujino, A. Borak, C. Falub, T. Fromherz, L. Diehl, H. Sigg, and D. Grutzmacher, Phys. Rev. B 72, 153315 (2005).

${ }^{14}$ V. J. Goldman, D. C. Tsui, and J. E. Cunningham, Phys. Rev. Lett. 58, 1256 (1987).

${ }^{15}$ H. Sunamura, N. Usami, Y. Shiraki, and S. Fukatsu, Appl. Phys. Lett. 66, 3024 (1995).

${ }^{16}$ P. Schittenhelm, M. Gail, J. Brunner, J. F. Nützel, and G. Abstreiter, Appl. Phys. Lett. 67, 1292 (1995).

${ }^{17}$ U. Gennser, M. Scheinert, L. Diehl, S. Tsujino, A. Borak, C. V. Falub, D. Grützmacher, A. Weber, D. K. Maude, G. Scalari, Y. Campidelli, O. Kermarrec, and D. Bensahel, Europhys. Lett. 74, 882 (2006).

${ }^{18}$ P. W. Lukey, J. Caro, L. J. Geerligs, K. Werner, and S. Radelaar, Microelectron. Eng. 27, 87 (1995).

${ }^{19}$ U. Gennser, V. P. Kesan, S. S. Iyer, J. Bucelot, and E. S. Yang, J. Vac. Sci. Technol. B 9, 2059 (1991).

${ }^{20} \mathrm{In}$ our samples, the barrier height is equal to $0.47 \mathrm{eV}$ for $\mathrm{HH}$ and $0.24 \mathrm{eV}$ for LH.

${ }^{21}$ G. D. Shen, D. X. Xu, M. Willander, and G. V. Hansson, Phys. Rev. B 45, 9424 (1992).

${ }^{22}$ L. Diehl, S. Mentese, E. Müller, D. Grützmacher, H. Sigg, U. Gennser, I. Sagnes, Y. Campidelli, O. Kermarrec, D. Bensahel, and J. Faist, Appl. Phys. Lett. 81, 4700 (2002).

${ }^{23}$ S. Tsujino, H. Sigg, M. Scheinert, D. Grützmacher, and J. Faist, IEEE J. Sel. Top. Quantum Electron. 12, 1642 (2006).

${ }^{24}$ J. P. A. van der Wagt, A. C. Seabaugh, and E. A. Beam, IEEE Electron Device Lett. 19, 7 (1998); Also, see the following and references therein, S. Tsujino, D. Grützmacher, and U. Gennser, in Silicon Heterostructure Handbook: Materials, Fabrication, Devices, Circuits, and Applications of SiGe and Si Strained-Layer Epitaxy, edited by J. D. Cressler (CRC, Boca Raton, FL, 2005), p. 647. 